\title{
Scopolamine and medial frontal stimulus-processing during interval timing
}

Qiang Zhang ${ }^{1}$, Dennis Jung ${ }^{1,2}$, Travis Larson ${ }^{1}$, Youngcho Kim ${ }^{1}$, Nandakumar S. Narayanan ${ }^{1, *}$

\section{Affiliations:}

1: Department of Neurology, Carver College of Medicine, University of Iowa, Iowa City, IA 52242

2: Present address: Neuroscience Graduate Program, University of Rochester, 601 Elmwood Avenue, Rochester, NY 14642

\section{*Corresponding Author}

Nandakumar Narayanan

319-353-5698

Nandakumar-narayanan@uiowa.edu

169 Newton Road

Pappajohn Biomedical Discovery Building - 1336

University of Iowa, Iowa City, 52242 


\section{Highlights}

- The cholinergic muscarinic inhibitor scopolamine impairs interval timing behavior.

- Scopolamine does not change time-related ramping activity in the medial frontal cortex.

- Medial prefrontal stimulus-related modulation increased 


\begin{abstract}
Neurodegenerative diseases such as Parkinson's disease (PD), dementia with Lewy Bodies (DLB), and Alzheimer's disease (AD) involve loss of cholinergic neurons in the basal forebrain. Here, we investigate how cholinergic dysfunction impacts the frontal cortex during interval timing, a process that can be impaired in $\mathrm{PD}$ and $\mathrm{AD}$ patients. Interval timing requires participants to estimate an interval of several seconds by making a motor response, and depends on the medial frontal cortex (MFC), which is richly innervated by basal forebrain cholinergic projections. Past work has shown that scopolamine, a muscarinic cholinergic receptor antagonist, reliably impairs interval timing. We tested the hypothesis that scopolamine would attenuate timerelated ramping, a key form of temporal processing in the MFC. We recorded neuronal ensembles from 8 mice during performance of a 12-s fixed-interval timing task, which was impaired by the administration of scopolamine. Consistent with past work, scopolamine impaired timing. To our surprise, we found that time-related ramping was unchanged, but stimulus-related activity was enhanced in the MFC. Principal component analyses revealed no consistent changes in time-related ramping components, but did reveal changes in higher components. Taken together, these data indicate that scopolamine changes stimulus-processing rather than temporal processing in the MFC. These data could help understand how cholinergic dysfunction affects cortical circuits in diseases such as PD, DLB, and AD.
\end{abstract}

Key words: Scopolamine, interval timing, medial frontal cortex, neuronal ensemble recording, cholinergic circuit dysfunction, Parkinson's disease, dementia with Lewy Bodies, Alzheimer's disease 


\section{Introduction}

Cholinergic dysfunction is a major feature of Alzheimer's disease (AD), Parkinson's disease (PD), and dementia with Lewy bodies (DLB) [1-5]. In particular, cholinergic neurons are located in the basal forebrain, which suffers marked neurodegeneration in AD, PD, and DLB [1, 6-9]. Furthermore, drugs that block acetylcholine breakdown, such as donepezil and rivastigmine, can improve cognitive function in $\mathrm{PD}, \mathrm{DLB}$, and $\mathrm{AD}$. Basal forebrain cholinergic neurons project broadly to the cortex; however, it is unknown how cholinergic dysfunction affects cortical circuits.

One cognitive process that depends on cholinergic circuits and is consistently impaired in $\mathrm{AD}$ and $\mathrm{PD}$ is interval timing, which requires subjects to estimate an interval of several seconds by making a motor response [10-13]. Interval timing is ideal for investigating cortical cholinergic function because 1) it depends on the medial frontal cortex (MFC), which is disrupted in AD, PD, and DLB [14-18], 2) it is highly conserved across mammalian species and thus can be readily investigated in rodent models [19], and 3) interval timing in rodents is reliably impaired when they are given scopolamine, a cholinergic inhibitor of M1 muscarinic receptors [20, 21]. Our recent work has indicated that a key form of temporal processing in the rodent MFC is timerelated ramping activity; in other words, monotonic increases or decreases in firing rate across a temporal interval $[16,22,23]$. These data lead to the specific hypothesis that scopolamine impairs time-related ramping by MFC neurons.

We tested this hypothesis by recording neuronal ensembles from mice performing a 12-s fixed-interval-timing task, and administering intraperitoneal saline or scopolamine. We found that while scopolamine impaired interval timing, it did not affect time-related ramping in the MFC. Surprisingly, it increased stimulus-related processing in this brain structure. We interpret these data in the context of cholinergic functions and circuits relevant for AD, PD, and DLB. 


\section{Experimental Procedures}

Mice: This study used 8 wild-type C57/BL6J male mice purchased from Jackson Laboratories (000664) at 3 months of age. Mice consumed 1-1.5 g of sucrose pellets during each behavioral session, and additional food was provided 1-2 $\mathrm{hr}$ after each behavioral session in the home cage. Single housing and a 12-hr light/dark cycle were used; all experiments took place during the light cycle. Mice were maintained at $80-85 \%$ of their baseline body weight during the course of these experiments for motivation. All procedures were approved by the Animal Care and Use Committee (\#707239) at the University of Iowa, in accordance with the National Institutes of Health Guide for the Care and Use of Laboratory Animals.

Mouse fixed-interval timing task: Mice were trained to perform an interval-timing task with a 12-sec interval[11]. Operant chambers (MedAssociates) were equipped with a nose poke hole with a yellow LED stimulus light (ENV-313W), a pellet dispenser (ENV-203-20), and a house light (ENV-315W). Behavioral chambers were housed in sound-attenuating chambers

(MedAssociates). All behavioral responses including nose pokes and access to pellet receptacles were recorded with infra-red sensors. First, animals learned to make operant nose pokes to receive rewards (20-mg rodent purified pellets, F0071, BioServe). After fixed-ratio training, animals were trained in a 12-sec fixed-interval timing task in which rewards were delivered for responses after a 12-sec interval (Figure 1A). The house light was turned on to signal the start of the 12 -sec interval. Early responses were not rewarded. Responses after $12 \mathrm{sec}$ resulted in trial termination with reward delivery. Rewarded nose pokes were signaled by the house light turning off. Each trial was followed by a $30 \pm 6$-sec pseudorandom inter-trial interval that concluded with the house light turning on, signaling the beginning of the next trial. All sessions were 60 min long. Responses were summed into time-response histograms with 1 -sec bins from 0 to 18 sec after trial start. For plotting, we used the MATLAB function ksdensity.m to estimate the probability density function time-response histograms with a bandwidth of 1 , normalized to maximum response rate, and averaged across animals. We quantified timing using a measure of the curvature of time-response histograms. This metric is based on the cumulative distribution function's deviation from a straight line; it is 0 when the time-response curve is flat during the interval but closer to 1 when more responses are at $12 \mathrm{~s}$ and time-response histograms are more curved. We and others have used this metric extensively to quantify timing because curvature is 
resistant to differences in overall response rate [11, 24-26]. Curvature indices and response rates were compared between the normal saline and the scopolamine groups using a two-tailed t-test; $\mathrm{p}<0.05$ was interpreted as statistically significant.

Surgical procedures: Mice trained in the 12-sec fixed-interval-timing task were implanted with recording microelectrode arrays (Microprobes) targeting the MFC prior to neurophysiology recordings. Briefly, mice were anesthetized using ketamine $(100 \mathrm{mg} / \mathrm{kg})$ and xylazine $(10 \mathrm{mg} / \mathrm{kg})$. A surgical level of anesthesia was maintained, with ketamine supplements $(10 \mathrm{mg} / \mathrm{kg})$ given hourly (or as needed) and regular monitoring for stable respiratory rate and absent toe pinch response. Mice were placed in the stereotactic equipment with non-rupturing ear bars. A heating pad was used to prevent hypothermia. Under aseptic surgical conditions, the skull was leveled between the bregma and lambda. A single craniotomy was drilled over the area above the MFC and three holes were drilled for skull screws. For recording experiments, animals were implanted (coordinates from the bregma: AP: $+1.8, \mathrm{ML}+0.5, \mathrm{DV}-1.8$ ) with a microelectrode array configured as a $4 \times 4$ array of $50 \mu \mathrm{m}$ stainless steel wires $(200 \mu \mathrm{m}$ between wires and rows; impedance measured in vitro at 400-600 k $\Omega$; Microprobes). Electrode ground wires were wrapped around the skull screws. The electrode array was inserted while concurrently recording neuronal activity. The craniotomy was sealed with cyanoacrylate ("SloZap”, Pacer Technologies) accelerated by “ZipKicker” (Pacer Technologies), and methyl methacrylate (“dental cement”; AM Systems). Following implantation, animals were allowed to recover for two weeks before being reacclimatized to behavioral and recording procedures.

Neuronal ensemble recordings: Freely moving electrophysiological recordings were performed as described in detail previously $[11,24]$. Following training in a 12-sec interval timing task and MFC implantation with 16-channel microelectrodes, mice were subjected to intraperitoneal injection with normal saline or scopolamine $(1 \mathrm{mg} / \mathrm{kg}$, Sigma-Aldrich, S0929) while being recorded during the 12 -sec interval timing task. Mice were connected to recording head stages and a cable without anesthesia. Neuronal ensemble recordings in the MFC were made using a multi-electrode recording system (Open Ephys). Raw wideband signal was high-pass filtered at $0.05 \mathrm{~Hz}$ with total gain of 5000, and recorded with 16-bit digitization at 30k Hz sampling rate. To detect spikes, raw signals were rereferenced using common median referencing to minimize potential non-neural electrical noise, and band-pass filtered between 300 and $6000 \mathrm{~Hz}$ offline. 
Spikes were detected with a threshold of 5 median absolute deviations. A Plexon Offline Sorter was used to sort single units and to remove artifacts. PCA and waveform shape were used for spike sorting. Single units were identified as having 1) consistent waveform shape, 2) separable clusters in PCA space, 3) a consistent refractory period of at least $1 \mathrm{~ms}$ in inter-spike-interval histograms, and 4) consistent firing rates around behavioral events. Unique neurons were verified by constructing two-dimensional cumulative distribution probabilities from Pearson's correlation coefficients of pair-wise waveform and inter-spike-interval comparisons, and using a one-tailed threshold of $\mathrm{p}<0.05$. Spike activity was analyzed for all cells that fired at rates above $0.1 \mathrm{~Hz}$. Local field potential (LFP) was recorded with bandpass filters between 0.05 and $1000 \mathrm{~Hz}$. Statistical summaries were based on all recorded neurons. No subpopulations were selected or filtered out of the neuron database. Analysis of neuronal activity and quantitative analysis of basic firing properties were carried out with custom routines for MATLAB (all raw data and MATLAB scripts are available at our lab website: https://narayanan.lab.uiowa.edu/ article/datasets). All behavioral events were recorded simultaneously using TTL inputs at 30k Hz. Peri-event rasters and average histograms were constructed around trial start.

We analyzed our neuronal data according to procedures described at length previously $[11,24]$. For each neuron, we constructed peri-event spike data from $-2 \mathrm{sec}$ to $12 \mathrm{sec}$ after trial start. As in the past, we defined time-related ramping neurons as those with a significant fit via linear regression of time vs. firing rate over the interval binned at $1 \mathrm{sec}$. Finally, we defined stimulus-modulated and response-modulated neurons as those with trial-by-trial changes in firing rate 0-200 msec after event onset compared to -500 to $-300 \mathrm{msec}$ prior to stimulus onset with a $\mathrm{p}<0.05$ via a paired t-test. Pearson's chi-squared test was used to compare the number of modulated neurons between saline and scopolamine sessions.

We analyzed neuronal patterns using PCA, which we have used to identify patterns of neuronal activity in an unbiased, data-driven manner [11, 27-29]. PCA was constructed from ztransformed peri-event time histograms over the entire interval binned at $0.1 \mathrm{sec}$ and smoothed with a gaussian kernel over 5 bins. All neurons from 8 mice from sessions with saline or scopolamine infusions were included in PCA. We then used a t-test to compare PCs between saline and scopolamine sessions. 
MFC LFP power was calculated in defined frequency bands (delta: 1-4 Hz, theta: 5-8 Hz; alpha: 9-12 Hz; beta: 13-30 Hz) during the interval (0-12 s) using wavelet-based time-frequency analyses.

Histology: When experiments were complete, mice were euthanized by injections of $100 \mathrm{mg} / \mathrm{kg}$ sodium pentobarbital. All mice were intracardially perfused with $4 \%$ paraformaldehyde. The brain was removed and post-fixed in paraformaldehyde overnight and immersed in $30 \%$ sucrose until the brains sank. Sections $(40 \mu \mathrm{m})$ were made on a cryostat (Leica) and stored in cryoprotectant (50\% PBS, 30\% ethylene glycol, $20 \%$ glycerol) at $-20^{\circ} \mathrm{C}$, before being mounted onto slides with mounting media containing DAPI (Invitrogen P36962). Images were captured on an Olympus VS120 Microscope. 


\section{Results}

\section{Scopolamine impairs fixed-interval timing}

Two past studies in rodents have demonstrated that scopolamine impairs interval timing [20, 21]. During neuronal recording sessions, we found that scopolamine infusion markedly changed timeresponse histograms during fixed-interval timing (Fig 1C). We calculated the curvature of timeresponse histograms to measure timing, a metric based on cumulative distribution functions that we and others have used in the past [11, 24-26]. We found that scopolamine significantly decreased the curvature of time-response histograms $(0.25+/-0.02$ vs. $0.03+/-0.03$ with scopolamine; paired $t_{(7)}=5.1, p=0.001$; Fig 1D). Furthermore, we noticed that there were more responses early in the interval and that scopolamine decreased the ratio of responses late in the interval vs. early in the interval (last $6 \mathrm{sec}$ divided by first $6 \mathrm{sec} ; 3.27$ +/- 0.31 vs. 1.60 +/- 0.26 with scopolamine, paired $t_{(7)}=3.9, p=0.006$; Fig 1E). Scopolamine did not change the number of overall responses between 0 and $12 \mathrm{sec}(86.3+/-23.0$ vs. $98.4+/-28.5$ with scopolamine $)$ or the number of rewards (66.6 +/- 5.3 vs. $63.8+/-5.3$ with scopolamine). Taken together, our findings are consistent with past work demonstrating that scopolamine impairs interval timing [20, 21].

\section{Scopolamine does not change MFC ramping but increases stimulus-related processing.}

Prior work by our group and others has demonstrated that time-related ramping activity by MFC neurons is a key correlate of temporal processing [16, 23]. As scopolamine impairs interval timing, we hypothesized that this drug would impair time-related ramping. We tested this idea by identifying time-related ramping MFC neurons by linear regression (Fig 2A), and comparing the number of MFC ramping neurons in sessions with saline and scopolamine. In 8 mice, of 117 MFC neurons recorded during saline sessions, 41 exhibited time-related ramping (35\%). Critically, a similar fraction of MFC neurons exhibited ramping with scopolamine (36 of 108, or $33 \%$ ); this did not support our hypothesis.

Next, we looked at other modulation patterns in MFC. Prefrontal regions can powerfully affect stimulus-processing [30-32]. During fixed-interval timing, this stimulus is a light that goes on at trial start (Fig 2B). Surprisingly, there were twice as many stimulus-modulated MFC neurons in scopolamine sessions (32 of 108, or 30\%) compared to saline sessions (16 of 117, or 
$\left.14 \% ; \mathrm{X}^{2}=7.6, \mathrm{p}=0.006\right)$. There were no differences in the number of response-modulated neurons (Figure 2C; 39\% with saline vs. 44\% with scopolamine). These data provide evidence that neither MFC ramping nor response-related activities are changed by scopolamine; by contrast, scopolamine increased MFC stimulus-related modulation.

When comparing average MFC neuronal ensemble activity in saline and scopolamine sessions, we noticed subtle differences late in the interval (increased activity at black arrow in Fig 3A vs. decreased activity at black arrow in Fig 3B). These resulted in different average activities of MFC neuronal ensembles with saline vs. scopolamine (Fig 3C). To capture these differences with data-driven techniques, we turned to principal component analysis, which have been used extensively to identify patterns in complex neuronal data[11, 27-29]. We found three common patterns. PC1, which explained $29 \%$ of variance, exhibited time-related ramping activity, consistent with extensive past work from our group (Fig 3D-E). Of note, this component did not change with scopolamine, consistent with our results above and contrary to our hypothesis. PC2, which explained $19 \%$ of variance, was broadly modulated across the interval, and also did not change with scopolamine. By contrast, PC3, which explained $12 \%$ of variance, had a more complex pattern, with a peak close to $7 \mathrm{sec}$ in the interval. Of note, in the scopolamine sessions there was a more negative score with PC3 compared to saline sessions (Fig $\left.3 \mathrm{~F}, \mathrm{PC} 3 ; \mathrm{t}_{(223)}=2.4, \mathrm{p}<0.02\right)$. These data provide further evidence that scopolamine did not change MFC ramping but could change more complex aspects of MFC neuronal ensemble activity.

Finally, we examined MFC LFP oscillatory power during the interval (0-12 s; Figure 4). We found no consistent changes in delta $(1-4 \mathrm{~Hz})$, theta $(5-8 \mathrm{~Hz})$, alpha $(9-12 \mathrm{~Hz})$, or beta activity (13-30 Hz). In summary, our results suggest that scopolamine impaired interval timing and enhances stimulus-related processing in MFC without changing MFC temporal processing or MFC LFPs. 


\section{Discussion}

We tested the hypothesis that scopolamine would attenuate time-related ramping activity in the MFC. We found no evidence that scopolamine changed MFC ramping by linear regression or PCA. To our surprise, we found that stimulus-related processing was increased in the MFC with scopolamine. These data provide insight into how scopolamine might influence cortical circuits during interval timing.

Many cognitive behaviors are impaired by the muscarinic cholinergic inhibitor scopolamine, often through attentional and stimulus-processing deficits [33, 34]. Scopolamine reliably causes timing deficits in rodents [20,21], and here we report similar deficits during fixed-interval timing. Surprisingly, MFC ramping is intact with scopolamine administration. There are two possibilities that might account for this result. First, we and others have identified neuronal ramping as a key mechanism of temporal processing in the MFC [16, 22, 23, 35], but it is possible that ramping is not explicitly related to timing. In this case, others have proposed temporal computations based on oscillatory activity [36], and we note that PC3 has oscillatory features, although the period appeared to be longer than 1 second. Scopolamine may affect neuronal oscillatory activity that was not detected by our analyses. A second possibility is that scopolamine affects stimulus-processing mechanisms in the MFC and this triggers animals to respond despite intact MFC temporal processing [31,37]. The increase in responses early in the interval is supportive of this idea.

Previous studies have shown that acetylcholine is crucial for stimulus processing. Microdialysis has revealed that acetylcholine is increased during tasks involving sustained attention, changes in ambient light, anticipation of a meal, motor activity, and handling, while amperometry has indicated that MFC acetylcholine can increase for reward-predictive cues [3843]. Acetylcholine can also be increased during attentionally demanding tasks such as 5-choice serial reaction-time tasks $[31,44]$. Lesioning of MFC cholinergic projections impairs the processing of fast but not slow stimuli, as well as causing marked deficits in visual attentional performance [45]. To our knowledge MFC cholinergic projections have never been studied in interval timing, although cholinergic projections to the visual cortex affect learning of temporal intervals but not interval-timing performance [46]. Lesioning of MFC cholinergic projections decreased stimulus-related activity and the performance of a visual attention task, contrary to our 
results here with scopolamine, a muscarinic antagonist [47]. In primates, cholinergic deafferentation can affect working memory, and cholinergic effects on working memory and attention occur as a result of the direct effects on stimulus processing [48, 49]. These studies broadly support a role for cortical cholinergic circuits in task-related stimulus processing.

Our results here are quite different from those for systemic or focal manipulations of dopaminergic circuits in the frontal cortex or striatum [12, 27, 50, 51]. While dopaminergic manipulations also reliably affect interval timing, our work indicates that manipulating prefrontal dopamine via D1-type dopamine receptors affects both time-related ramping activity and $\sim 4 \mathrm{~Hz}$ rhythms $[11,27]$. Scopolamine appears to have distinct effects on MFC circuits enhancing stimulus-related activity while leaving MFC ramping and MFC LFPs unchanged. These data suggest that cholinergic and dopaminergic manipulations have distinct and specific effects on cortical circuits. Future studies with cell-type specific resolution might be able to further resolve themes of cortical cholinergic vs. dopaminergic circuits.

Patients with AD and PD have deficits in timing [10, 11, 15, 52-54]. We are not aware of studies of interval timing in DLB patients. PD and AD have prominent cholinergic deficits [1,2]. Of note, cholinergic drugs can improve cognition in patients with AD, PD and DLB [55]. However, little is known about the relevant cholinergic circuit mechanisms of these effects.

Our study has several limitations. First, scopolamine was administered systemically and is a poor model of cognitive dysfunction in dementia, as it can act on all muscarinic acetylcholine receptors in the brain [33] and other brain systems[56]. Furthermore, it can have non-specific locomotor and autonomic effects, although we did not observe an increased response rate and observed highly specific neuronal effects on stimulus-processing in this study. Cholinergic interneurons in the striatum or the MFC may also be critical mediators of cognitive processing [57]. Finally, we are unsure if scopolamine directly modulates MFC stimulus-related neurons or modulates other brain areas. Nevertheless, our results lay important groundwork for highly specific investigation of cholinergic circuits using cell-type specific methods in future work.

In conclusion, we performed neuronal ensemble recording from the MFC of freely moving rodents, and found that muscarinic cholinergic inhibition caused timing deficits and 
hyper-stimulus-modulation during interval timing. MFC temporal processing was not significantly affected. These results are consistent with the consensus that acetylcholine plays major roles in attention, working memory and stimulus processing, while the dopaminergic system is crucial for the neuronal "ramping" activities and an internal pacemaker.

Neuromodulation therapies targeting the cholinergic circuits have potential as treatments for AD as well as DLB [55]. Data from this study will help us understand the cholinergic circuits and may have relevance for diseases involving cholinergic deficits, such as AD and DLB.

\section{Contributors:}

QZ, YK and NN designed the study. QZ, DJ, and TL performed the experiments and collected the data. QZ, YK and NN analyzed the data. QZ, YK and NN interpreted the results and wrote the manuscript.

\section{Acknowledgements:}

NN is supported by R01MH116043A1. QZ is supported by the NINDS R25 grant, a pilot project grant from the Aging Mind and Brain Initiative at University of Iowa, and the physician scientist training program at University of Iowa. QZ is a trainee of the University of Iowa Clinical Neuroscientist Training Program (CNS-TP).

\section{Conflicts of interests:}

There are no conflicts of interests. 


\section{Figures and legends:}
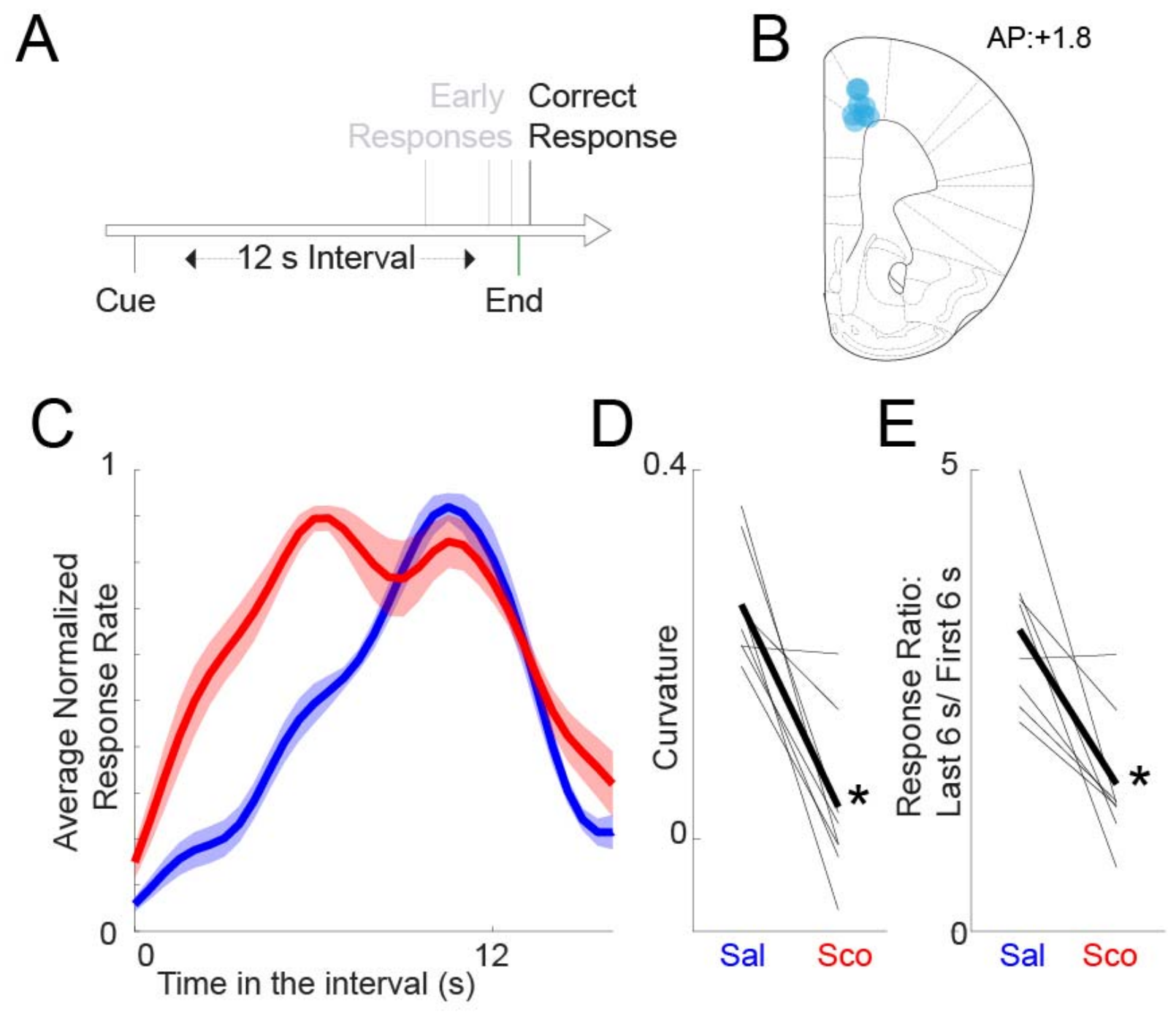

Figure 1. Scopolamine impairs interval timing. A) Interval timing task: Mice were trained to perform a fixed-interval timing task with a $12 \mathrm{~s}$ interval. The first response after $12 \mathrm{~s}$ led to a food reward; early responses were unreinforced. B) Recording electrode locations (blue dots) in the medial frontal cortex. C) Time-response histograms during fixed-interval timing. Data for mice administered saline are plotted in blue, and those for mice administered scopolamine are plotted in red. D) Timing can be quantified by computing a curvature index of time-response 
histograms; scopolamine decreases the curvature index, indicating flatter time-response histograms. E) We also noticed that animals with scopolamine responded more during the earlier portion of the interval. The ratio of responses in the last 6 vs. first 6 s closer to one, unlike in sessions with saline injected. Data from 8 mice; $*=p<0.05$ via paired t-test. 

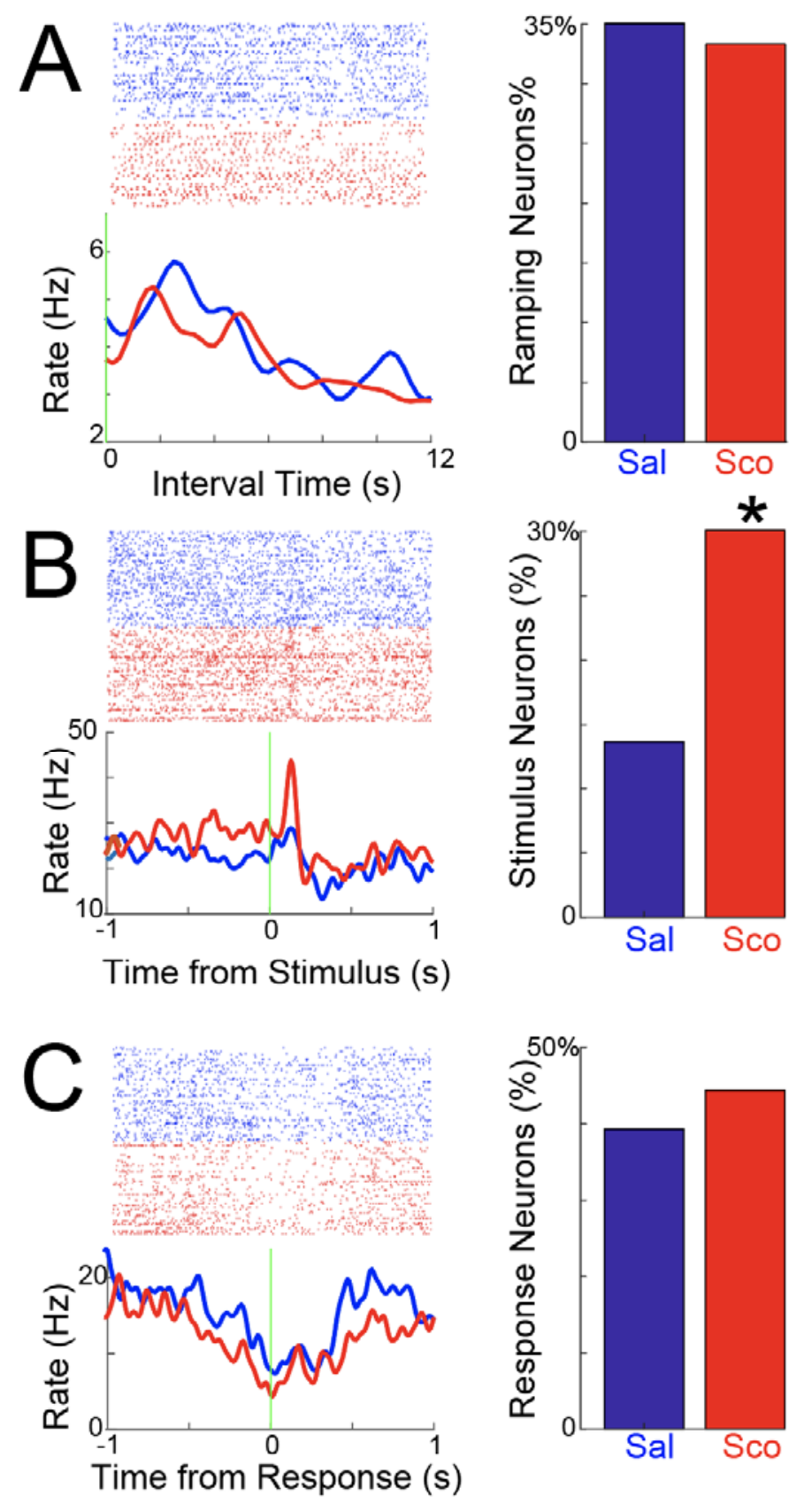

Figure 2. Scopolamine does not change MFC time-related ramping, but increases MFC stimulus-related activity. A) We identified MFC neurons with time-related ramping activity by linear regression. Crucially, scopolamine did not change the number of MFC ramping neurons. B) By contrast, scopolamine dramatically increased the number of MFC neurons with stimulusrelated modulation. C) Scopolamine did not change MFC neurons with response-related modulation. Data from 8 mice; 117 MFC neurons recorded in saline sessions and 108 neurons recorded in scopolamine sessions; $*=p<0.05$ via $\chi^{2}$ test. 

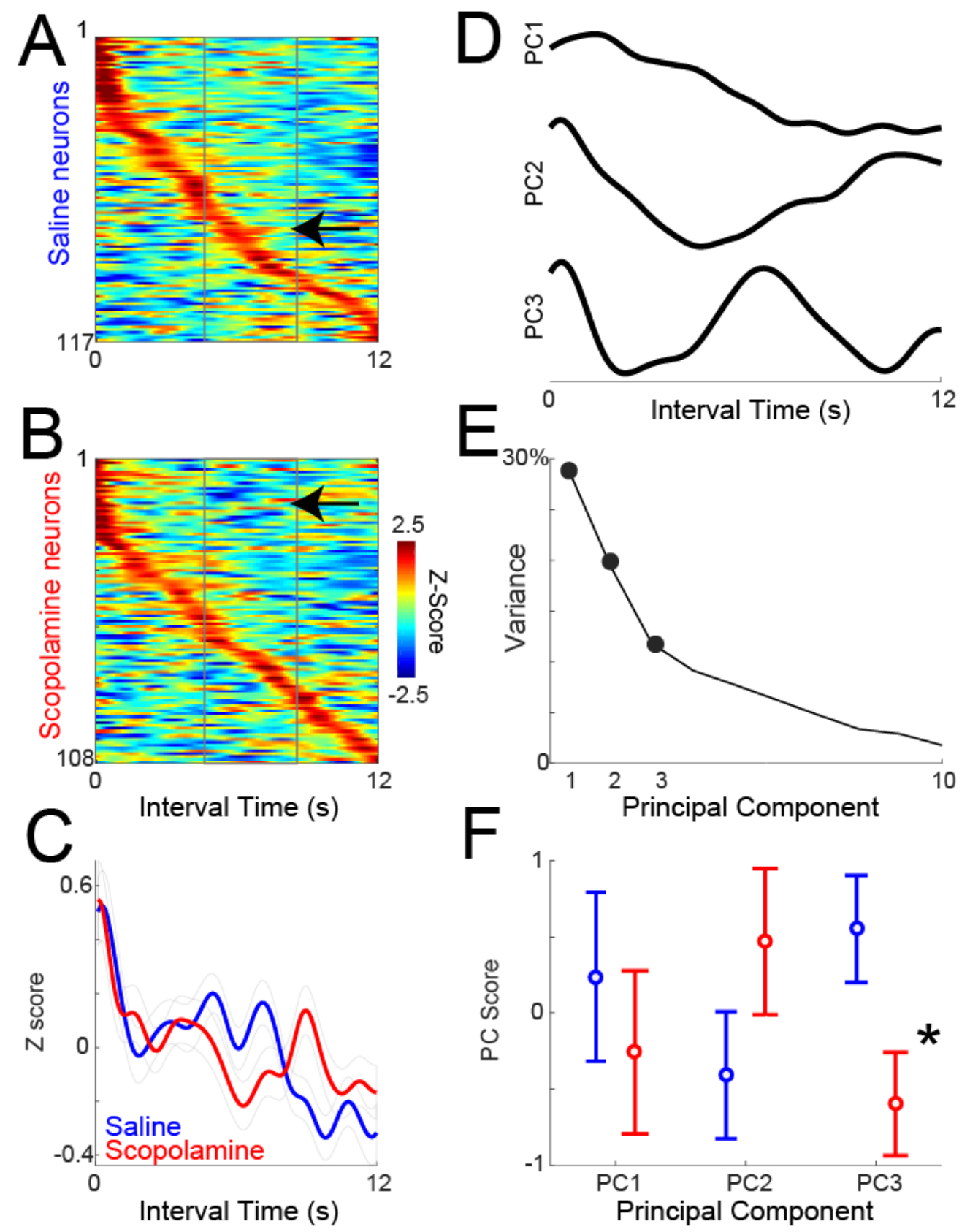

Figure 3. Neuronal ensemble effects of scopolamine: A) Average Z-scored neuronal activity during the interval shown for all MFC neurons treated with saline, and B) scopolamine, sorted by peak activity. We noticed subtle differences in activity, with more activity in saline (arrow in A), vs. less activity in scopolamine (arrow in B) late in the interval. Activity binned at $10 \mathrm{~ms}$ and smoothed over 5 bins. C) There were differences during the interval between average MFC neuronal activities in saline vs. scopolamine sessions. D) To quantify these differences using data-driven techniques, we turned to principal component analysis, which identified 3 major 
patterns. PC1 had ramping patterns, PC2 was modulated during the interval, and PC3 had more complex modulation. E) Fraction of variance explained by each component. F) Only PC3 was different between saline and scopolamine sessions. Data from 8 mice; 117 MFC neurons recorded in saline sessions and 108 neurons recorded in scopolamine sessions; $*=\mathrm{p}<0.05$ via ttest. 

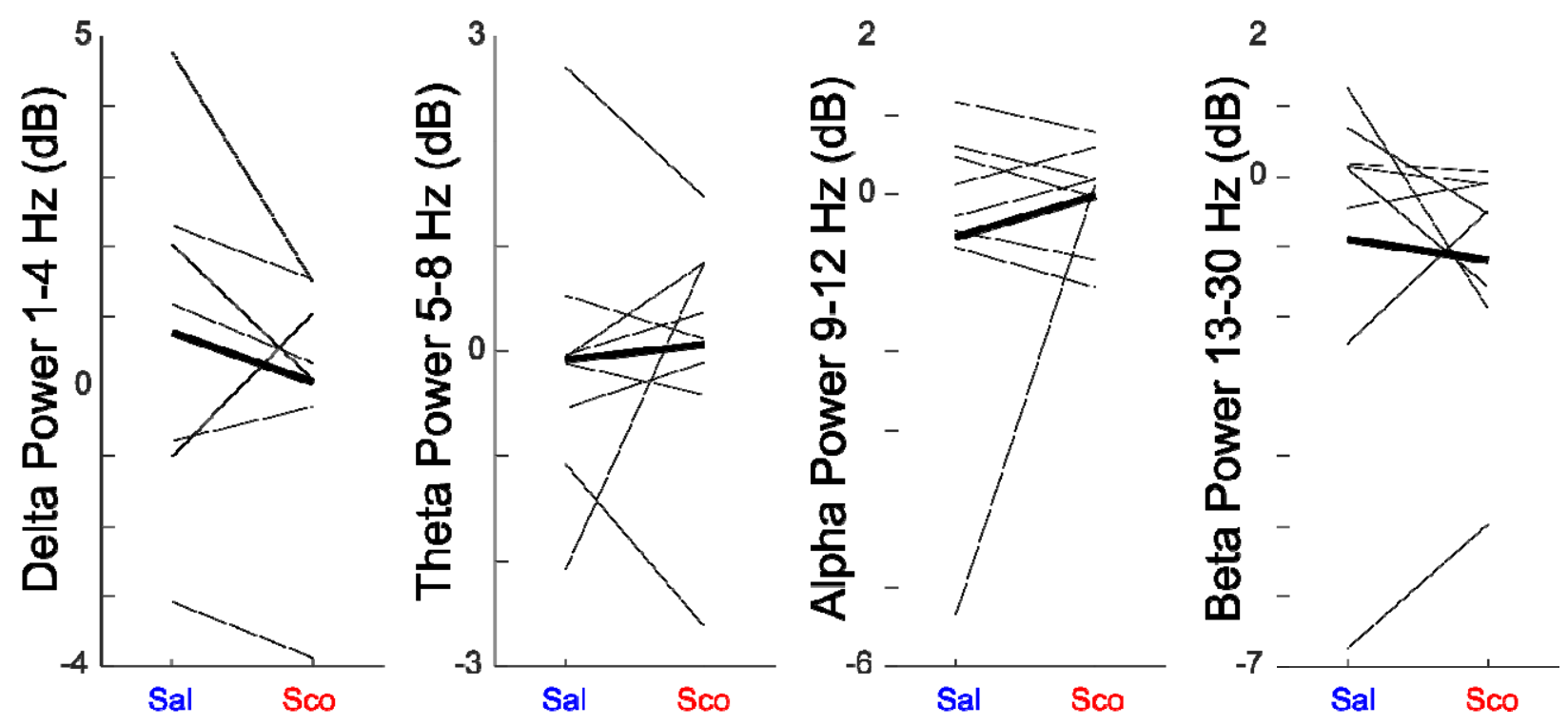

Figure 4. Scopolamine does not change MFC LFP activity: We measured MFC LFPs from 8 mice in delta, theta, alpha, and beta power in saline and scopolamine sessions. We did not observe consistent differences between saline and scopolamine sessions. Data from 8 mice. 


\section{References}

[1] T. Arendt, V. Bigl, A. Arendt, A. Tennstedt, Loss of neurons in the nucleus basalis of Meynert in Alzheimer's disease, paralysis agitans and Korsakoff's Disease, Acta Neuropathol, 61 (1983) 101-108. [2] P. Tiraboschi, L.A. Hansen, M. Alford, M.N. Sabbagh, B. Schoos, E. Masliah, L.J. Thal, J. Corey-Bloom, Cholinergic dysfunction in diseases with Lewy bodies, Neurology, 54 (2000) 407-411.

[3] N.I. Bohnen, R.L. Albin, The cholinergic system and Parkinson disease, Behav Brain Res, 221 (2011) 564-573.

[4] N.I. Bohnen, D.I. Kaufer, R. Hendrickson, L.S. Ivanco, B.J. Lopresti, G.M. Constantine, A. Mathis Ch, J.G. Davis, R.Y. Moore, S.T. Dekosky, Cognitive correlates of cortical cholinergic denervation in Parkinson's disease and parkinsonian dementia, J Neurol, 253 (2006) 242-247.

[5] N.I. Bohnen, D.I. Kaufer, L.S. Ivanco, B. Lopresti, R.A. Koeppe, J.G. Davis, C.A. Mathis, R.Y. Moore, S.T. DeKosky, Cortical cholinergic function is more severely affected in parkinsonian dementia than in Alzheimer disease: an in vivo positron emission tomographic study, Arch Neurol, 60 (2003) 1745-1748. [6] M.V. Johnston, M. McKinney, J.T. Coyle, Evidence for a cholinergic projection to neocortex from neurons in basal forebrain, Proc Natl Acad Sci U S A, 76 (1979) 5392-5396.

[7] V. Bigl, N.J. Woolf, L.L. Butcher, Cholinergic projections from the basal forebrain to frontal, parietal, temporal, occipital, and cingulate cortices: a combined fluorescent tracer and acetylcholinesterase analysis, Brain Res Bull, 8 (1982) 727-749.

[8] P.J. Whitehouse, J.C. Hedreen, C.L. White, 3rd, D.L. Price, Basal forebrain neurons in the dementia of Parkinson disease, Ann Neurol, 13 (1983) 243-248.

[9] J.P. Gratwicke, T. Foltynie, Early nucleus basalis of Meynert degeneration predicts cognitive decline in Parkinson's disease, Brain, 141 (2018) 7-10.

[10] L. Caselli, L. Iaboli, P. Nichelli, Time estimation in mild Alzheimer's disease patients, Behav Brain Funct, 5 (2009) 32.

[11] Y.C. Kim, S.W. Han, S.L. Alberico, R.N. Ruggiero, B. De Corte, K.H. Chen, N.S. Narayanan, Optogenetic Stimulation of Frontal D1 Neurons Compensates for Impaired Temporal Control of Action in DopamineDepleted Mice, Curr Biol, 27 (2017) 39-47.

[12] K.L. Parker, K.H. Chen, J.R. Kingyon, J.F. Cavanagh, N.S. Narayanan, Medial frontal approximately 4$\mathrm{Hz}$ activity in humans and rodents is attenuated in PD patients and in rodents with cortical dopamine depletion, J Neurophysiol, 114 (2015) 1310-1320.

[13] C. Malapani, B. Rakitin, R. Levy, W.H. Meck, B. Deweer, B. Dubois, J. Gibbon, Coupled temporal memories in Parkinson's disease: a dopamine-related dysfunction, J Cogn Neurosci, 10 (1998) 316-331.

[14] K.L. Parker, S.L. Alberico, A.D. Miller, N.S. Narayanan, Prefrontal D1 dopamine signaling is necessary for temporal expectation during reaction time performance, Neuroscience, 255 (2013) 246-254.

[15] K.L. Parker, D. Lamichhane, M.S. Caetano, N.S. Narayanan, Executive dysfunction in Parkinson's disease and timing deficits, Front Integr Neurosci, 7 (2013) 75.

[16] E.B. Emmons, B.J. De Corte, Y. Kim, K.L. Parker, M.S. Matell, N.S. Narayanan, Rodent Medial Frontal Control of Temporal Processing in the Dorsomedial Striatum, J Neurosci, 37 (2017) 8718-8733.

[17] J. Kim, A.H. Jung, J. Byun, S. Jo, M.W. Jung, Inactivation of medial prefrontal cortex impairs time interval discrimination in rats, Front Behav Neurosci, 3 (2009) 38.

[18] J.T. Coull, R.K. Cheng, W.H. Meck, Neuroanatomical and neurochemical substrates of timing, Neuropsychopharmacology, 36 (2011) 3-25.

[19] C.V. Buhusi, W.H. Meck, What makes us tick? Functional and neural mechanisms of interval timing, Nat Rev Neurosci, 6 (2005) 755-765.

[20] W.H. Meck, Neuropharmacology of timing and time perception, Brain Res Cogn Brain Res, 3 (1996) 227-242. 
[21] F. Balci, E.A. Ludvig, J.M. Gibson, B.D. Allen, K.M. Frank, B.J. Kapustinski, T.E. Fedolak, D. Brunner, Pharmacological manipulations of interval timing using the peak procedure in male $\mathrm{C} 3 \mathrm{H}$ mice, Psychopharmacology (Berl), 201 (2008) 67-80.

[22] P. Simen, F. Balci, L. de Souza, J.D. Cohen, P. Holmes, A model of interval timing by neural integration, J Neurosci, 31 (2011) 9238-9253.

[23] N.S. Narayanan, Ramping activity is a cortical mechanism of temporal control of action, Curr Opin Behav Sci, 8 (2016) 226-230.

[24] Y.C. Kim, N.S. Narayanan, Prefrontal D1 Dopamine-Receptor Neurons and Delta Resonance in Interval Timing, Cereb Cortex, DOI 10.1093/cercor/bhy083(2018).

[25] W. Fry, R.T. Kelleher, L. Cook, A mathematical index of performance on fixed-interval schedules of reinforcement, J Exp Anal Behav, 3 (1960) 193-199.

[26] N.S. Narayanan, B.B. Land, J.E. Solder, K. Deisseroth, R.J. DiLeone, Prefrontal D1 dopamine signaling is required for temporal control, Proc Natl Acad Sci U S A, 109 (2012) 20726-20731.

[27] K.L. Parker, K.H. Chen, J.R. Kingyon, J.F. Cavanagh, N.S. Narayanan, D1-dependent $4 \mathrm{~Hz}$ oscillations and ramping activity in rodent medial frontal cortex during interval timing, J Neurosci, 34 (2014) 1677416783.

[28] N.S. Narayanan, M. Laubach, Delay activity in rodent frontal cortex during a simple reaction time task, J Neurophysiol, 101 (2009) 2859-2871.

[29] J.K. Chapin, M.A. Nicolelis, Principal component analysis of neuronal ensemble activity reveals multidimensional somatosensory representations, J Neurosci Methods, 94 (1999) 121-140.

[30] S. Liebe, G.M. Hoerzer, N.K. Logothetis, G. Rainer, Theta coupling between V4 and prefrontal cortex predicts visual short-term memory performance, Nat Neurosci, 15 (2012) 456-462, S451-452.

[31] F. Passetti, Y. Chudasama, T.W. Robbins, The frontal cortex of the rat and visual attentional performance: dissociable functions of distinct medial prefrontal subregions, Cereb Cortex, 12 (2002) $1254-1268$.

[32] T.P. Zanto, M.T. Rubens, A. Thangavel, A. Gazzaley, Causal role of the prefrontal cortex in top-down modulation of visual processing and working memory, Nat Neurosci, 14 (2011) 656-661.

[33] I. Klinkenberg, A. Blokland, The validity of scopolamine as a pharmacological model for cognitive impairment: a review of animal behavioral studies, Neurosci Biobehav Rev, 34 (2010) 1307-1350.

[34] M. Sarter, J.P. Bruno, Cognitive functions of cortical acetylcholine: toward a unifying hypothesis, Brain Res Brain Res Rev, 23 (1997) 28-46.

[35] J. Wang, D. Narain, E.A. Hosseini, M. Jazayeri, Flexible timing by temporal scaling of cortical responses, Nat Neurosci, 21 (2018) 102-110.

[36] M.S. Matell, W.H. Meck, Cortico-striatal circuits and interval timing: coincidence detection of oscillatory processes, Brain Res Cogn Brain Res, 21 (2004) 139-170.

[37] K.R. Daffner, M.M. Mesulam, L.F. Scinto, D. Acar, V. Calvo, R. Faust, A. Chabrerie, B. Kennedy, P. Holcomb, The central role of the prefrontal cortex in directing attention to novel events, Brain, 123 ( Pt 5) (2000) 927-939.

[38] J. Day, G. Damsma, H.C. Fibiger, Cholinergic activity in the rat hippocampus, cortex and striatum correlates with locomotor activity: an in vivo microdialysis study, Pharmacol Biochem Behav, 38 (1991) 723-729.

[39] F.M. Inglis, H.C. Fibiger, Increases in hippocampal and frontal cortical acetylcholine release associated with presentation of sensory stimuli, Neuroscience, 66 (1995) 81-86.

[40] A.M. Himmelheber, M. Sarter, J.P. Bruno, Operant performance and cortical acetylcholine release: role of response rate, reward density, and non-contingent stimuli, Brain Res Cogn Brain Res, 6 (1997) 2336. 
[41] A.M. Himmelheber, J. Fadel, M. Sarter, J.P. Bruno, Effects of local cholinesterase inhibition on acetylcholine release assessed simultaneously in prefrontal and frontoparietal cortex, Neuroscience, 86 (1998) 949-957.

[42] J.P. Bruno, C. Gash, B. Martin, A. Zmarowski, F. Pomerleau, J. Burmeister, P. Huettl, G.A. Gerhardt, Second-by-second measurement of acetylcholine release in prefrontal cortex, Eur J Neurosci, 24 (2006) 2749-2757.

[43] V. Parikh, M. Sarter, Cholinergic mediation of attention: contributions of phasic and tonic increases in prefrontal cholinergic activity, Ann N Y Acad Sci, 1129 (2008) 225-235.

[44] F. Passetti, J.W. Dalley, M.T. O'Connell, B.J. Everitt, T.W. Robbins, Increased acetylcholine release in the rat medial prefrontal cortex during performance of a visual attentional task, Eur J Neurosci, 12 (2000) 3051-3058.

[45] B.D. Winters, T.W. Robbins, B.J. Everitt, Selective cholinergic denervation of the cingulate cortex impairs the acquisition and performance of a conditional visual discrimination in rats, Eur J Neurosci, 19 (2004) 490-496.

[46] A.A. Chubykin, E.B. Roach, M.F. Bear, M.G. Shuler, A cholinergic mechanism for reward timing within primary visual cortex, Neuron, 77 (2013) 723-735.

[47] T.M. Gill, M. Sarter, B. Givens, Sustained visual attention performance-associated prefrontal neuronal activity: evidence for cholinergic modulation, J Neurosci, 20 (2000) 4745-4757.

[48] M.L. Furey, P. Pietrini, J.V. Haxby, W.C. Drevets, Selective effects of cholinergic modulation on task performance during selective attention, Neuropsychopharmacology, 33 (2008) 913-923.

[49] P.L. Croxson, D.A. Kyriazis, M.G. Baxter, Cholinergic modulation of a specific memory function of prefrontal cortex, Nat Neurosci, 14 (2011) 1510-1512.

[50] K.L. Parker, R.N. Ruggiero, N.S. Narayanan, Infusion of D1 Dopamine Receptor Agonist into Medial Frontal Cortex Disrupts Neural Correlates of Interval Timing, Front Behav Neurosci, 9 (2015) 294.

[51] B.J. De Corte, L.M. Wagner, M.S. Matell, N.S. Narayanan, Striatal dopamine and the temporal control of behavior, Behav Brain Res, 356 (2019) 375-379.

[52] M.C. Carrasco, M.J. Guillem, R. Redolat, Estimation of short temporal intervals in Alzheimer's disease, Exp Aging Res, 26 (2000) 139-151.

[53] C. Papagno, A. Allegra, M. Cardaci, Time estimation in Alzheimer's disease and the role of the central executive, Brain Cogn, 54 (2004) 18-23.

[54] A.D. Rueda, M. Schmitter-Edgecombe, Time estimation abilities in mild cognitive impairment and Alzheimer's disease, Neuropsychology, 23 (2009) 178-188.

[55] Q. Zhang, Y.C. Kim, N.S. Narayanan, Disease-modifying therapeutic directions for Lewy-Body dementias, Front Neurosci, 9 (2015) 293.

[56] S.L. Alberico, Y.C. Kim, T. Lence, N.S. Narayanan, Axial levodopa-induced dyskinesias and neuronal activity in the dorsal striatum, Neuroscience, 343 (2017) 240-249.

[57] I.B. Witten, S.C. Lin, M. Brodsky, R. Prakash, I. Diester, P. Anikeeva, V. Gradinaru, C. Ramakrishnan, K. Deisseroth, Cholinergic interneurons control local circuit activity and cocaine conditioning, Science, 330 (2010) 1677-1681. 
A

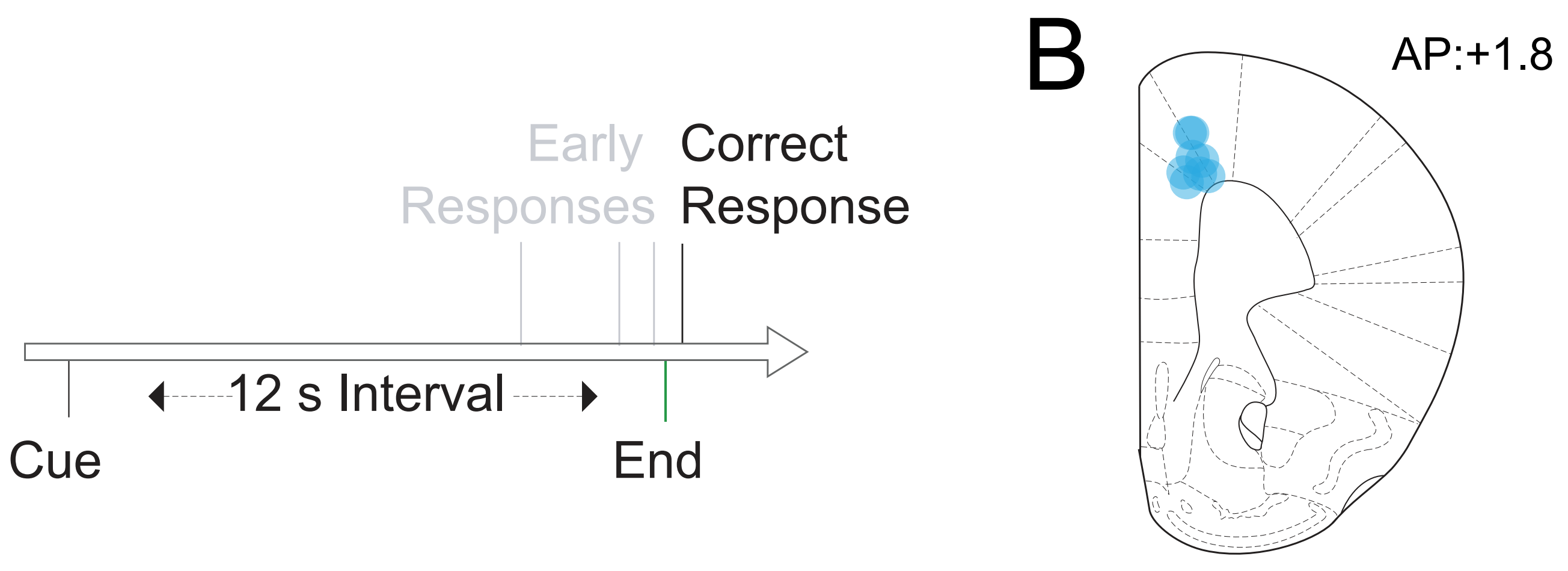

C

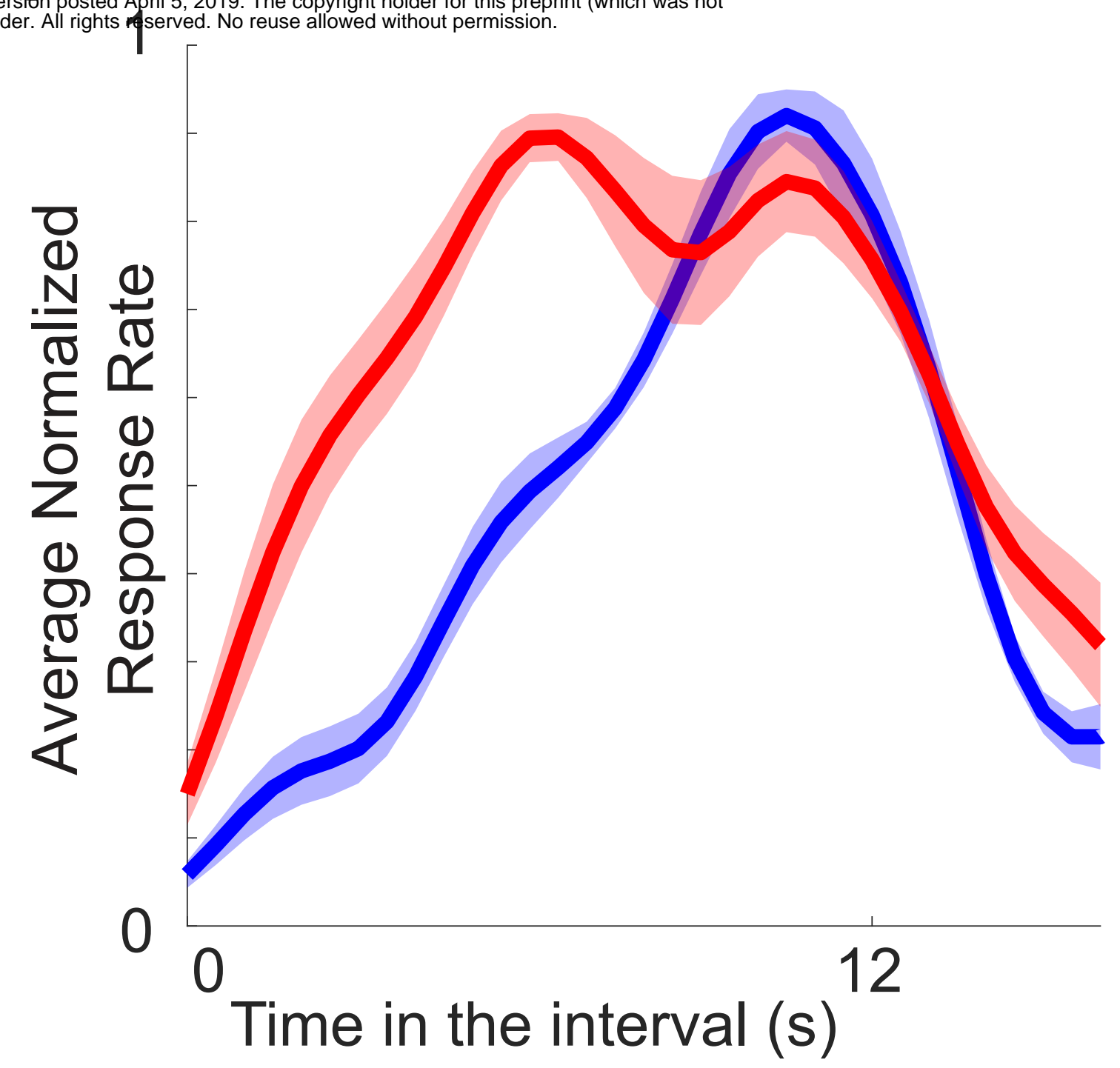

D

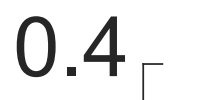

$E_{5}$

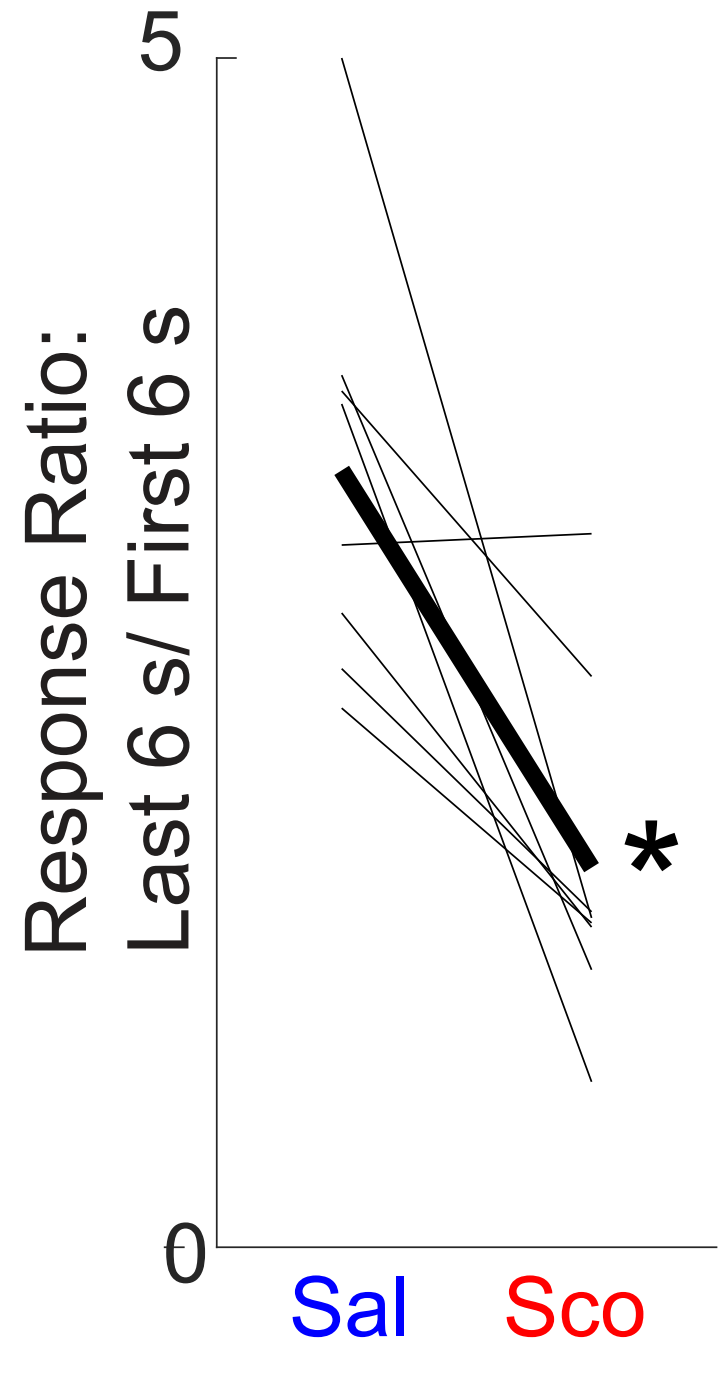




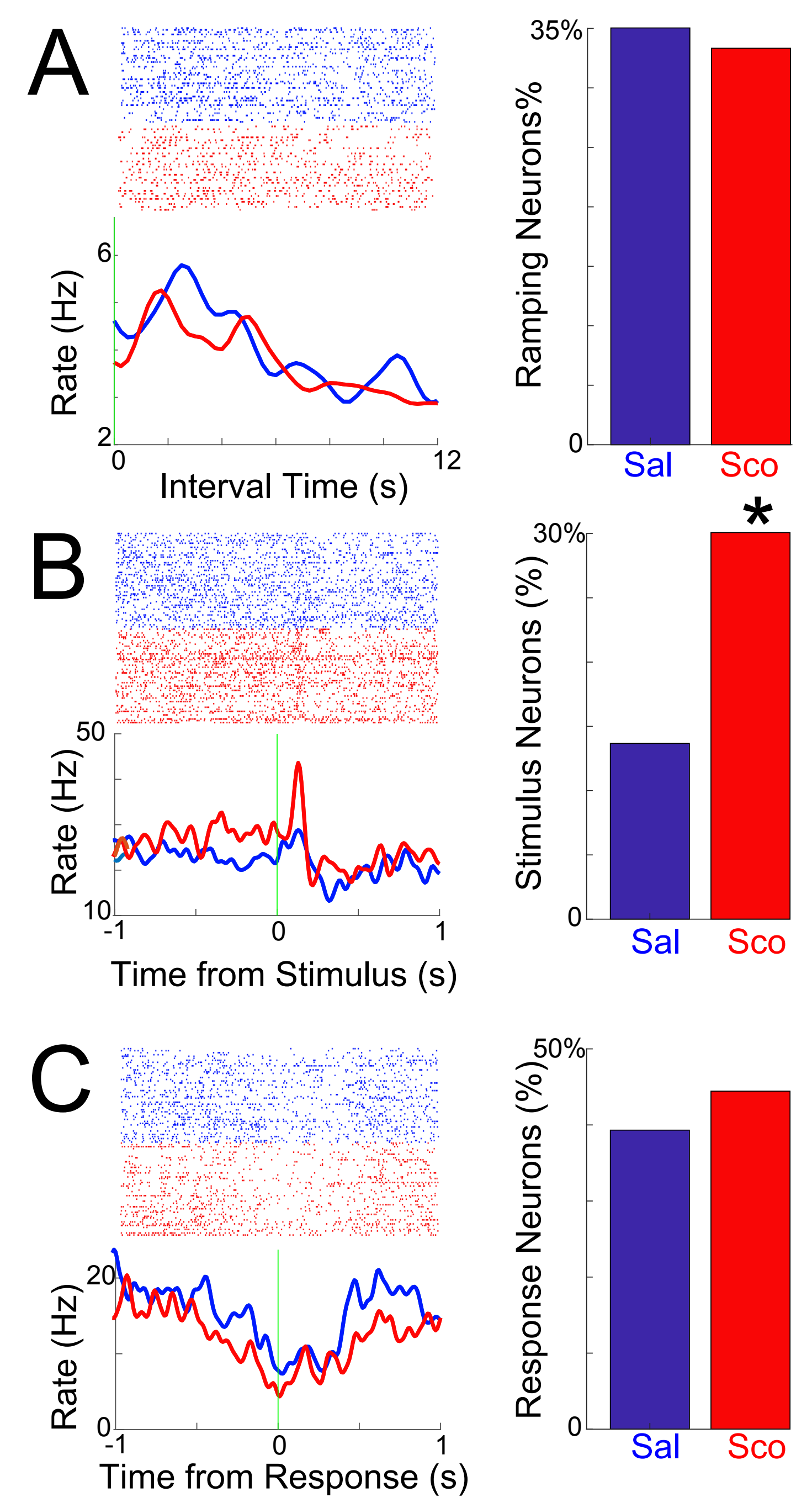



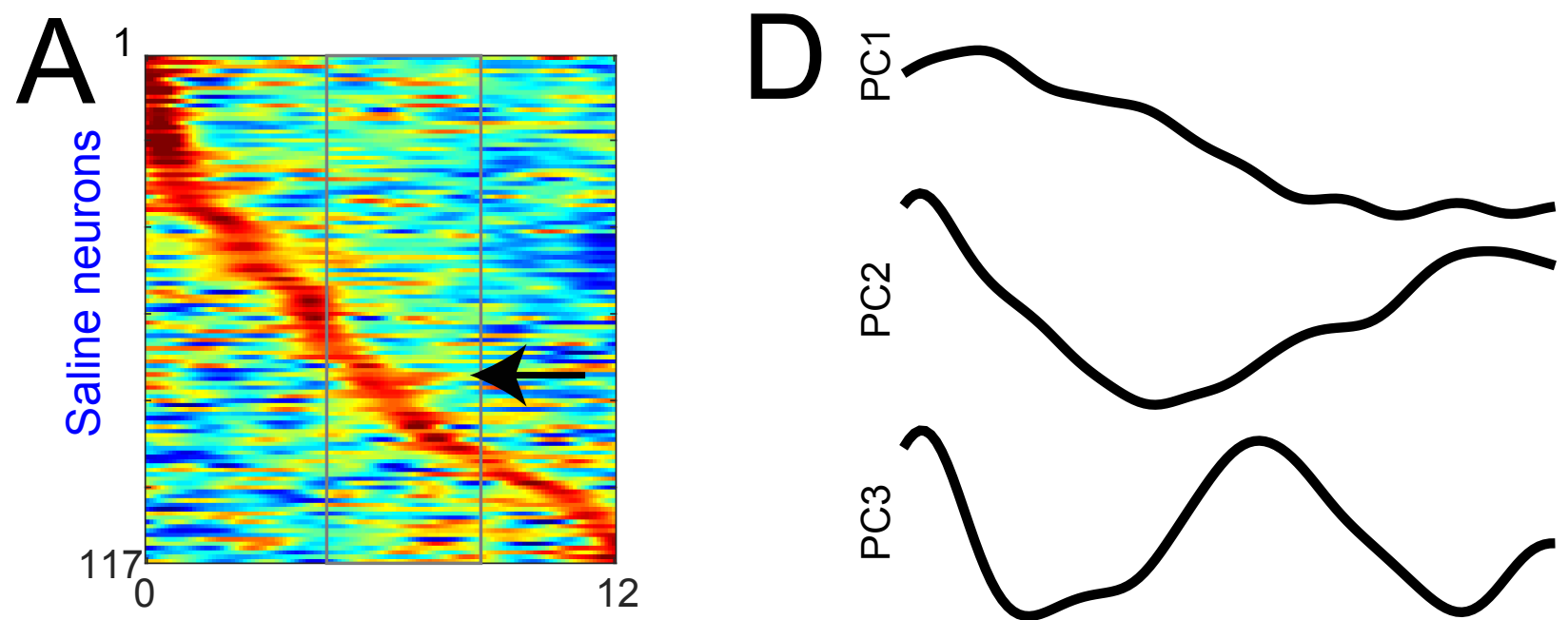

$D$
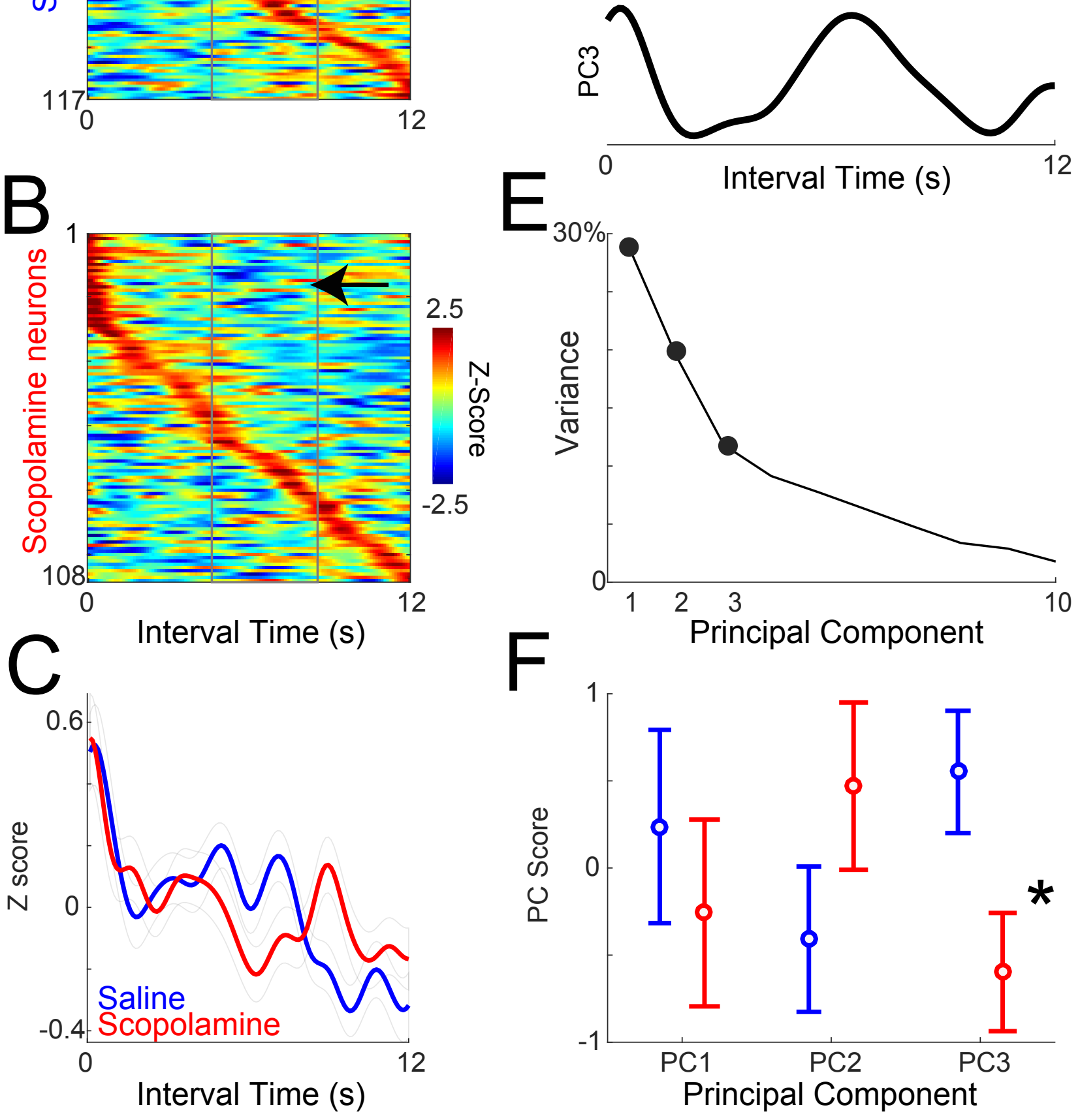

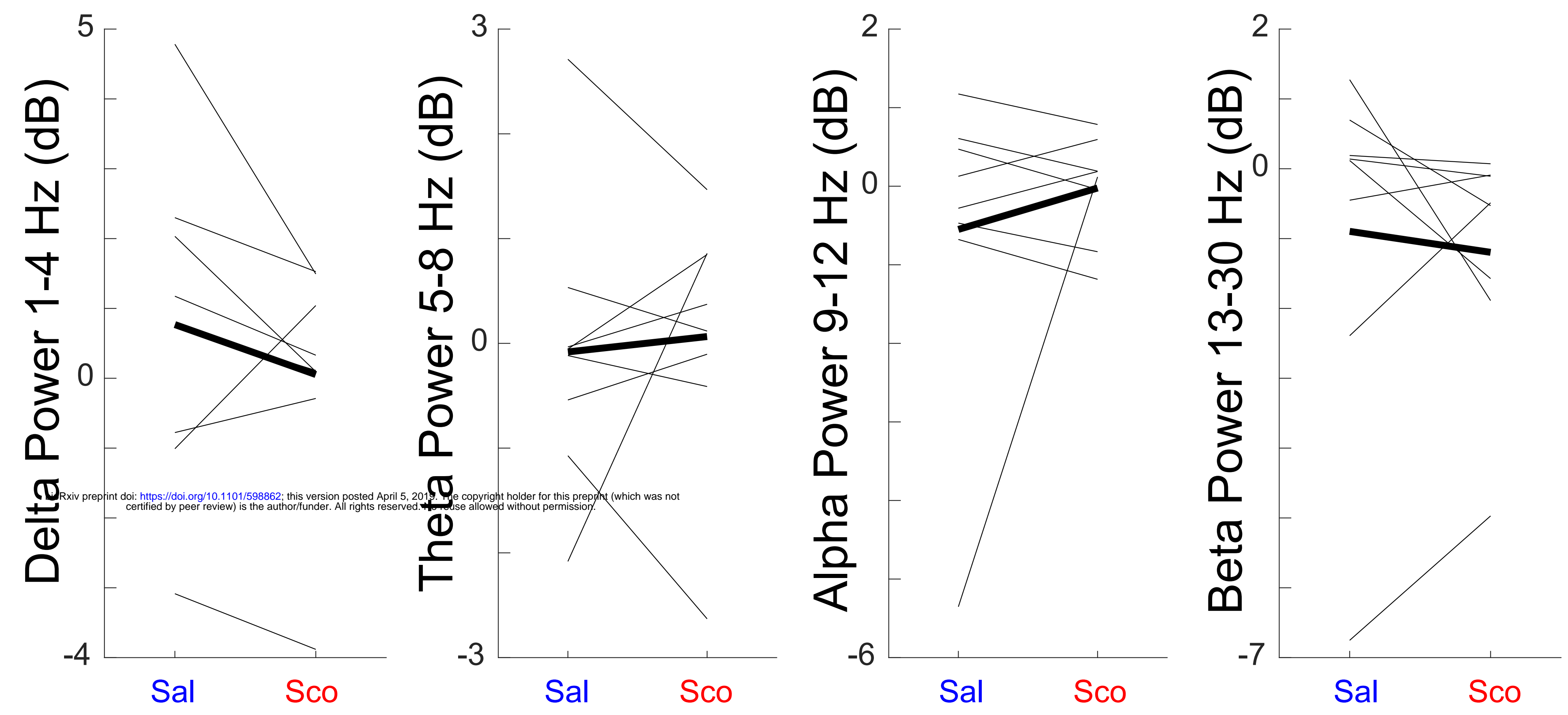